\title{
DUALITIES FOR SUBRESIDUATED LATTICES
}

\author{
CELANI SERGIO A., NAGY AGUSTÍN L. AND SAN MARTÍN HERNÁN J.
}

\begin{abstract}
A subresiduated lattice is a pair $(A, D)$, where $A$ is a bounded distributive lattice, $D$ is a bounded sublattice of $A$ and for every $a, b \in A$ there is $c \in D$ such that for all $d \in D, d \wedge a \leq b$ if and only if $d \leq c$. This $c$ is denoted by $a \rightarrow b$. This pair can be regarded as an algebra $\langle A, \wedge, \vee, \rightarrow, 0,1\rangle$ of type $(2,2,2,0,0)$ where $D=\{a \in A: 1 \rightarrow a=a\}$. The class of subresiduated lattices is a variety which properly contains to the variety of Heyting algebras.

In this paper we present dual equivalences for the algebraic category of subresiduated lattices. More precisely, we develop a spectral style duality and a bitopological style duality for this algebraic category. Finally we study the connections of these results with a known Priestley style duality for the algebraic category of subresiduated lattices.
\end{abstract}

\section{INTRODUCTION}

In this paper we are interested in the study of subresiduated lattices [4, 7], which are a generalization of Heyting algebras [1]. A subresiduated lattice [7] is a pair $(A, D)$, where $A$ is a bounded distributive lattice, $D$ is a bounded sublattice of $A$ and for every $a, b \in A$ there is $c \in D$ such that for all $d \in D, d \wedge a \leq b$ if and only if $d \leq c$. This $c$ is denoted by $a \rightarrow b^{1}$. This pair can be regarded as an algebra $\langle A, \wedge, \vee, \rightarrow, 0,1\rangle$ of type $(2,2,2,0,0)$ where $D=\{a \in A: 1 \rightarrow a=a\}$. The class of subresiduated lattices properly contains to the variety of Heyting algebras. It follows from [7, Theorem 1] that an algebra $\langle A, \wedge, \vee, \rightarrow, 0,1\rangle$ is a subresiduated lattice if and only if $\langle A, \wedge, \vee, 0,1\rangle$ is a bounded distributive lattice and the following conditions are satisfied for every $a, b, c \in A:(a \wedge b) \rightarrow a=1, a \rightarrow b \leq c \rightarrow(a \rightarrow b)$, $a \wedge(a \rightarrow b) \leq b$ and $c \rightarrow(a \wedge b)=(c \rightarrow a) \wedge(c \rightarrow b)$. Thus, the class of subresiduated lattices forms a variety. A different equational base for this variety was given in [4], where this variety is presented as a subvariety of the variety of weak Heyting algebras.

Subresiduated lattices were introduced by Epstein and Horn in [7] with the aim to study certain propositional logics defined in a language without classical implication but with a connective of implication which is called strict implication. The logics studied in [7] are examples of subuintuitionistic logics, i.e., logics in the language of intuitionistic logic that are defined semantically by using Kripke models, in the same way as intuitionistic logic is defined, but without requiring of the models some of the properties required in the intuitionistic case [3, 4]. Recall that S4-algebras are Boolean algebras with a modal operator $\square$ in the language that satisfies the identities $\square(1)=1, \square(a \wedge b)=\square(a) \wedge \square(b), \square(a) \leq a$ and $\square(a) \leq \square(\square(a))$. It is known that the variety $\mathrm{S} 4$, whose members are the S4-algebras, is the algebraic semantic of the modal logic $\mathbf{S} 4$. This means that $\phi$ is a theorem of $\mathbf{S} 4$ if and only if the variety S4 satisfies $\phi \approx 1$. The variety of subresiduated lattices corresponds

\footnotetext{
${ }^{1}$ The bounded distributive lattice $D$ endowed with the binary operation $\rightarrow$ is a Heyting algebra.
} 
to the variety of algebras defined for all the equations $\phi \approx 1$ satisfied in the variety S4 where only appears the connectives conjunction $\wedge$, disjunction $\vee$, bottom $\perp$, top $T$ and a new connective of implication (called strict implication) defined by $\varphi \Rightarrow \psi:=\square(\varphi \rightarrow \psi)$, where $\rightarrow$ denotes the classical implication. The logic (considered as a set of theorems) which corresponds to the variety of subresiduated lattices is an example of subuintuitionistic logic. Analogously, the variety of Heyting algebras is the subvariety of the variety of subresiduated lattices which corresponds to the intuitionistic logic.

Topological dualities provide a very useful tool for the study of algebraic logic. In [11] Stone proved that there is a dual equivalence between the algebraic category BDL of bounded distributive lattices and the category Spec of what later became known as spectral spaces and spectral maps. In [9] Priestley described another dual categorical equivalence for BDL by means of ordered topological spaces known as Priestley spaces, thus establishing that BDL is also dually equivalent to the category PS of Priestley spaces and continuous order preserving maps. Since BDL is dually equivalent to both Spec and PS, it follows that the categories Spec and PS are equivalent. In fact, we can say more: Spec is actually isomorphic to PS (see for instance $[5,8]$ ). In $[2]$ the authors proved that $\mathrm{BDL}$ is also dually equivalent to a category of bitopological spaces and bicontinuous maps. Moreover, they proved that this category is isomorphic to PS and Spec.

In lattice theory there are many different dualities for algebraic categories whose objects have as reducts bounded distributive lattices. In particular, in $[4$, Subsection 4.1] it was given a Priestley-style duality for the algebraic category of subresiduated lattices. Our main goal is to build dual equivalences for the algebraic category of subresiduated lattices: a spectral style duality and a bitopological style duality.

The paper is organized as follows. In Section 2 we give some preliminaries and basic results. In particular, we give algebraic preliminaries about subresiduated lattices and topological preliminaries concerning dualities in lattice theory. In Section 3 we define the p-spectral spaces (Definition 3.1) and we show that in the associated spectral space of each subresiduated lattice (obtained via the spectral duality) it is possible to define, with the implication of the algebra, a preorder relation so that the spectral space endowed with such preorder relation is a p-spectral space. Conversely, we show that in the bounded distributive lattice associated to each p-spectral space (also obtained via the spectral duality) it is possible to define a binary operation, by using the preorder relation, so that this bounded distributive lattice endowed with such binary operation is a subresiduated lattice. Moreover, we extend these results in order to obtain a spectral style duality between the algebraic category of subresiduated lattices (named SRL) and a category whose objects are p-spectral spaces (named SSpec). In Section 4 we define the subresiduated bitopological spaces (Definition 4.1) and for every subresiduated lattice we build up a bitopological space on the set of its prime filters, which is also a subresiduated bitopological space. The connection between both topologies of any subresiduated bitopological space is the topological translation of the fact that subresiduated lattices can be defined as pairs $(A, D)$. Conversely, we show that for every subresiduated bitopological space we can build up a subresiduated lattice, where the implication is defined by using both topologies. Moreover, we extend these results in order to obtain a bitopological style duality between SRL and a category whose objects are the subresiduated bitopological spaces (named BS). Finally in Section 
5 we prove that the categories SSpec, BS and SRLS are isomorphic, where SRLS is the category given in [4] dually equivalent to SRL (see [4, Subsection 4.1]).

We give a table with some of the categories we shall consider in this paper:

\begin{tabular}{c|c|c} 
Category & Objects & Morphisms \\
\hline & Bounded distributive lattices & Algebra homomorphisms \\
SRL & Subresiduated lattices & Algebra homomorphisms \\
PS & Priestley spaces & Continuous order preserving maps \\
Spec & Spectral spaces & Spectral maps \\
SRLS & pWH-spaces & pWH-morphisms \\
SSpec & p-spectral spaces & p-spectral maps \\
BS & Subresiduated bitopological spaces & Subresiduated bitopological morphisms
\end{tabular}

The dual categorical equivalence for SRL considered in this manuscript can be summarized in the following diagram of categories and functors:

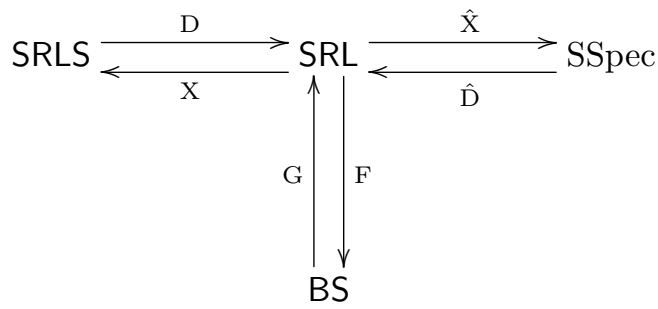

\section{Preliminaries and Basic Results}

In this section we give some preliminaries we shall use throughout the paper.

Let $(X, \leq)$ be a poset and $U \subseteq X$. We say that $U$ is an upset if for every $x, y \in X$, if $x \in U$ and $x \leq y$ then $y \in U$. Dually, we say that $U$ is a downset if for every $x, y \in X$, if $y \in U$ and $x \leq y$ then $x \in U$. The family of upsets of $(X, \leq)$ will be denoted by $\operatorname{Up}(X)$.

Let $X$ be a set and $U \subseteq X$. We define $U^{c}:=\{x \in X: x \notin U\}$. Let $S \subseteq X \times X$. We also define

$$
S(U)=\{x \in X:(y, x) \in S \text { for some } y \in U\}
$$

In particular, for $x \in X$ we write $S(x)$ in place of $S(\{x\})$, i.e.,

$$
S(x)=\{y \in X:(x, y) \in S\} .
$$

We also define

$$
S^{-1}(U)=\{x \in X: S(x) \cap U \neq \emptyset\} .
$$

Let $X$ be a set. For every $U, V \subseteq X$ we define the following subset of $X$ :

$$
U \Rightarrow_{S} V:=\{x \in X: S(x) \cap U \subseteq V\} .
$$

If there is not ambiguity or confusion we write $\Rightarrow$ in place of $\Rightarrow_{S}$. Notice that

$$
(U \Rightarrow V)^{c}=S^{-1}\left(U \cap V^{c}\right) .
$$

Let $X, Y, Z$ be sets, $R \subseteq X \times Y$ and $S \subseteq Y \times Z$. We define $R \circ S \subseteq X \times Z$ in the following way: $(x, z) \in R \circ S$ if and only if there exists $y \in Y$ such that $(x, y) \in R$ and $(y, z) \in S$. A WH-frame is a structure $(X, \leq, S)$ where $(X, \leq)$ is a poset and $S$ is a binary relation satisfying the condition $(\leq \circ S) \subseteq S$. 
2.1. Algebraic preliminaries. In what follows we recall definitions and properties involving bounded distributive lattices $[1,9]$ and subresiduated lattices $[4,7]$.

Let $A$ be a bounded distributive lattice. A subset $F$ of $A$ is said to be a filter if $1 \in F, F$ is an upset, and $a \wedge b \in F$ whenever $a, b \in F$. In particular, $F$ is said to be a prime filter if $F$ is a filter such that $F \neq A$ and for every $a, b \in A$, if $a \vee b \in F$ then $a \in F$ or $b \in F$. We write $X(A)$ for the set of prime filters of $A$. The map $\varphi_{A}: A \rightarrow \mathrm{Up}(X(A))$ given by

$$
\varphi_{A}(a)=\{P \in X(A): a \in P\}
$$

is a monomorphism of bounded distributive lattices [9]. If there is not ambiguity or confusion we write $\varphi$ in place of $\varphi_{A}$.

The following definition is [4, Definition 3.1].

Definition 2.1. A weak Heyting algebra, or WH-algebra for short, is an algebra $\langle A, \wedge, \vee, \rightarrow, 0,1\rangle$ of type $(2,2,2,0,0)$ such that $\langle A, \wedge, \vee, 0,1\rangle$ is a bounded distributive lattice and the following conditions are satisfied for every $a, b, c \in A$ :

(1) $(a \rightarrow b) \wedge(a \rightarrow c)=a \rightarrow(b \wedge c)$,

(2) $(a \rightarrow c) \wedge(b \rightarrow c)=(a \vee b) \rightarrow c$,

(3) $(a \rightarrow b) \wedge(b \rightarrow c) \leq a \rightarrow c$,

(4) $a \rightarrow a=1$

Let $\langle A, \wedge, \vee, \rightarrow, 0,1\rangle$ be a WH-algebra. If there is not ambiguity we write $A$ instead of $\langle A, \wedge, \vee, \rightarrow, 0,1\rangle$. Note that for every $a, b, c \in A$, if $a \leq b$ then $c \rightarrow a \leq$ $c \rightarrow b$ and $b \rightarrow c \leq a \rightarrow c$.

The following result follows from [4, Lemma 3.10].

Lemma 2.2. Let $(X, \leq, S)$ be a WH-frame. Then $\langle\operatorname{Up}(X), \cap, \cup, \Rightarrow, \emptyset, X\rangle$ is a WHalgebra.

Given a WH-algebra $A$ we define the binary relation $S_{A}$ on $\mathrm{X}(\mathrm{A})$ by

$$
(P, Q) \in S_{A} \Leftrightarrow \text { For every } a, b \in A \text {, if } a \rightarrow b \in P \text { and } a \in Q \text { then } b \in Q .
$$

It follows from $\left[4\right.$, Proposition 3.12] that $\left(X(A), \subseteq, S_{A}\right)$ is a WH-frame. Hence, $\mathrm{Up}(X(A))$ is a WH-algebra.

The following representation theorem is [4, Theorem 3.14].

Theorem 2.3. Let $A$ be a WH-algebra. Then the map $\varphi: A \rightarrow \operatorname{Up}(X(A))$ is a monomorphism.

The following result follows from [7, Theorem 1] and the footnote ${ }^{3)}$ of [4].

Proposition 2.4. Let $\langle A, \wedge, \vee, \rightarrow, 0,1\rangle$ be an algebra of type $(2,2,2,0,0)$. Then $\langle A, \wedge, \vee, \rightarrow, 0,1\rangle$ is a subresiduated lattice if and only if $\langle A, \wedge, \vee, \rightarrow, 0,1\rangle$ is a WHalgebra such that for every $a, b, c \in A, a \wedge(a \rightarrow b) \leq b$ and $a \rightarrow b \leq c \rightarrow(a \rightarrow b)$.

We write $\mathrm{SRL}$ for the variety of subresiduated lattices. If $A \in \mathrm{SRL}$ and $a, b \in A$, we have that

$$
1 \rightarrow(a \rightarrow b)=1 \wedge(1 \rightarrow(a \rightarrow b) \leq a \rightarrow b \leq 1 \rightarrow(a \rightarrow b) .
$$

Thus,

$$
1 \rightarrow(a \rightarrow b)=a \rightarrow b
$$


Hence, in every subresiduated lattice the equation $1 \rightarrow(a \rightarrow b)=a \rightarrow b$ is verified. The variety of Heyting algebras ${ }^{2}$ is a proper subvariety of the variety of SRL and SRL is a proper subvariety of the variety of WH-algebras (see for instance [10]).

A binary relation on a set is said to be a preorder if it is reflexive and transitive. Let $(X, \leq, S)$ be a WH-frame. Straightforward computations show that if $S$ is a reflexive relation then $U \cap(U \Rightarrow V) \subseteq V$ for every $U, V \in \operatorname{Up}(X)$ and that if $S$ is transitive relation then $U \Rightarrow V \subseteq W \Rightarrow(U \Rightarrow V)$ for every $U, V, W \in \operatorname{Up}(X)$. Thus, the following result follows from Lemma 2.2.

Proposition 2.5. Let $(X, \leq, S)$ be a WH-frame such that $S$ is a preorder. Then $\mathrm{Up}(X)$ is a subresiduated lattice.

Moreover, it holds the following result (which is [4, Proposition 4.17]).

Lemma 2.6. Let $A$ be a WH-algebra. Then

1) $a \wedge(a \rightarrow b) \leq b$ for every $a, b \in A$ if and only if $S_{A}$ is reflexive.

2) $a \rightarrow b \leq c \rightarrow(a \rightarrow b)$ for every $a, b, c \in A$ if and only if $S_{A}$ is transitive.

Now we use Proposition 2.5 in order to give an example of a subresiduated lattice which is not a Heyting algebra.

Example 1. Let $X$ be a set with two elements $x$ and $y$ and consider the poset $(X, \leq)$, where $\leq$ is defined by the equality relation, so the upsets of $(X, \leq)$ are $0:=\emptyset, a:=\{x\}, b:=\{y\}$ and $1:=X$. Also define $S=\{(x, x),(y, x),(y, y)\}$.

A straightforward computation shows that $(X, \leq, S)$ is a WH-frame such that $S$ is a preorder. Thus, it follows from Proposition 2.5 that $\operatorname{Up}(X)$ is a subresiduated lattice by considering the implication defined as $U \Rightarrow V=\{z \in X: S(z) \cap U \subseteq V\}$ for every $U, V \in \mathrm{Up}(X)$. Then we have the following table:

\begin{tabular}{c|cccc}
$\Rightarrow$ & 0 & $a$ & $b$ & 1 \\
\hline 0 & 1 & 1 & 1 & 1 \\
$a$ & 0 & 1 & 0 & 1 \\
$b$ & $a$ & $a$ & 1 & 1 \\
1 & 0 & $a$ & 0 & 1
\end{tabular}

Note that since $1 \Rightarrow b \neq b,\langle\operatorname{Up}(X), \cap, \cup, \Rightarrow, \emptyset, X\rangle$ is not a Heyting algebra ${ }^{3}$.

Now we give another characterization for subresiduated lattices.

Proposition 2.7. Let $A$ be a WH-algebra. Then the following conditions are equivalent:

(1) $A$ is a subresiduated lattice.

(2) For all $P, Q \in X(A),(P, Q) \in S_{A}$ if only if $P \cap D \subseteq Q$.

Proof. (1) $\Rightarrow(2)$ Let $A \in \mathrm{SRL}$ and $P, Q \in X(A)$. Suppose that $(P, Q) \in S_{A}$. Let $a \in P \cap D$. Since $a=1 \rightarrow a$ then $1 \rightarrow a \in P$. Taking into account that $1 \in Q$ we obtain that $a \in Q$. Hence, $P \cap D \subseteq Q$. Conversely, suppose that $P \cap D \subseteq Q$. Let $a, b \in A$ such that $a \rightarrow b \in P$ and $a \in Q$. Since $a \rightarrow b \in D$ then $a \rightarrow b \in P \cap D \subseteq Q$. Using that $a, a \rightarrow b \in Q$ we deduce that $a \wedge(a \rightarrow b) \in Q$. But $a \wedge(a \rightarrow b) \leq b$. so $b \in Q$. Thus, $(P, Q) \in S_{A}$.

The implication $(2) \Rightarrow(1)$ follows from Lemma 2.6.

\footnotetext{
${ }^{2}$ Given $A \in \mathrm{SRL}$ we have that $A$ is a Heyting algebra if and only if $b \leq a \rightarrow b$ for every $a, b \in A$.

${ }^{3}$ This subresiduated lattice can be also seen as the pair $(A, D)$, where $A$ is the bounded distributive lattice associated to $\operatorname{Up}(X)$ and $D$ is its bounded sublattice given by $D=\{0, a, 1\}$.
} 
Finally notice that it follows from [4, Proposition 4.20] and [4, Proposition 4.22] that if $A$ is a WH-algebra then $A$ is a Heyting algebra if and only if $S_{A}=\subseteq$.

2.2. Topological Preliminaries. In this section we give some topological results we shall use later. We recall Priestley duality [9], the spectral duality and a Priestley-style duality for the algebraic category of subresiduated lattices [4].

We start by recalling the Priestley duality [9]. A Priestley space is a triple $(X, \leq, \tau)$ such that $(X, \leq)$ is a poset, $(X, \tau)$ is a compact topological space and the Priestley separation axiom is satisfied, i.e., for every $x, y \in X$, if $x \not \leq y$ then there exists a clopen upset $U$ such that $x \in U$ and $y \notin U$. The set $\left\{U \cap V^{c}\right.$ : $U, V$ are clopen upsets $\}$ is a base of $(X, \tau)$. We write PS for the category whose objects are Priestley spaces and whose morphisms are the continuous order preserving maps. We write $\mathrm{BDL}$ for the algebraic category of bounded distributive lattices. If $A \in \mathrm{BDL}$ then $\mathrm{X}(A):=\left(X(A), \subseteq, \tau_{A}\right)$ is a Priestley space, where $\tau_{A}$ is the topology generated by the subbase $\{\varphi(a): a \in A\} \cup\left\{\varphi(a)^{c}: a \in A\right\}$. If $f: A_{1} \rightarrow A_{2}$ is a morphism in $\mathrm{BDL}$ then $\mathrm{X}(f): \mathrm{X}\left(A_{2}\right) \rightarrow \mathrm{X}\left(A_{1}\right)$ given by $\mathrm{X}(f)(P):=f^{-1}(P)$ is a morphism in PS. Moreover, the assignment $\mathrm{X}: \mathrm{BDL} \rightarrow \mathrm{PS}$ is a contravariant functor. If $(X, \leq, \tau) \in \mathrm{PS}$ then $\mathrm{D}(X):=(D(X), \cap, \cup, \emptyset, X) \in \mathrm{BDL}$, where $D(X)$ denotes the set of clopen upsets of $(X, \leq, \tau)$. If $g:\left(X_{1}, \leq_{1}, \tau_{1}\right) \rightarrow\left(X_{2}, \leq_{2}, \tau_{2}\right)$ is a morphism in PS then $\mathrm{D}(g): \mathrm{D}\left(X_{2}\right) \rightarrow \mathrm{D}\left(X_{1}\right)$ given by $\mathrm{D}(g)(U):=g^{-1}(U)$ is a morphism in BDL. Moreover, the assignment $\mathrm{D}: \mathrm{PS} \rightarrow \mathrm{BDL}$ is a contravariant functor. If $(X, \leq, \tau) \in \mathrm{PS}$ then the map $\epsilon_{X}:(X, \leq, \tau) \rightarrow \mathrm{X}(\mathrm{D}(X))$ given by $\epsilon_{X}(x)=\{U \in D(X): x \in U\}$ is an isomorphism in PS. If there is not ambiguity we write $\epsilon$ in place of $\epsilon_{X}$. If $A \in \mathrm{BDL}$ we also write $\varphi$ for the map $\varphi: A \rightarrow \mathrm{D}(\mathrm{X}(A))$ defined as in (2.1).

Proposition 2.8. The contravariant functors $\mathrm{X}: \mathrm{BDL} \rightarrow \mathrm{PS}$ and $\mathrm{D}: \mathrm{PS} \rightarrow \mathrm{BDL}$ define a dual categorical equivalence with natural isomorphisms $\varphi$ and $\epsilon$.

Now we recall the spectral duality. Let $(X, \tau)$ be a topological space. We say that a subset $C$ of $X$ is irreducible if for every $C_{1}$ and $C_{2}$ closed sets, if $C=C_{1} \cup C_{2}$ then $C=C_{1}$ or $C=C_{2}$. We say that $(X, \tau)$ is sober if every closed irreducible subset of $X$ is the closure of a point ${ }^{4}$. We write $\operatorname{KO}(X, \tau)$ for the set of compact open subsets of $X$. We say that $(X, \tau)$ is coherent if $\operatorname{KO}(X, \tau)$ is a basis for the topology. A spectral space is a topological space $(X, \tau)$ which is compact, coherent, sober and $\mathrm{KO}(X, \tau)$ is closed under finite intersections. If $f:\left(X_{1}, \tau_{1}\right) \rightarrow\left(X_{2}, \tau_{2}\right)$ is a function between coherent spaces such that $f^{-1}(U) \in \mathrm{KO}\left(X_{1}, \tau_{1}\right)$ for every $U \in \mathrm{KO}\left(X_{2}, \tau_{2}\right)$ we say that $f$ is a spectral function. We write Spec for the category whose objects are spectral spaces and whose morphism are the spectral functions between spectral spaces. If $A \in \mathrm{BDL}$ then $\hat{\mathrm{X}}(A):=\left(X(A), \hat{\tau}_{A}\right) \in$ Spec, where $\hat{\tau}_{A}$ is the topology with base $\{\varphi(a): a \in A\}$. If $f: A_{1} \rightarrow A_{2}$ is a morphism in BDL then $\hat{\mathrm{X}}(f): \hat{\mathrm{X}}\left(A_{2}\right) \rightarrow \hat{\mathrm{X}}\left(A_{1}\right)$ given by $\hat{\mathrm{X}}(f):=f^{-1}(P)$ is a morphism in Spec. If $(X, \tau) \in$ Spec then $\hat{\mathrm{D}}(X)=\langle\mathrm{KO}(X, \tau), \cap, \cup, \emptyset, X\rangle \in \mathrm{BDL}$. If $g:\left(X_{1}, \tau_{1}\right) \rightarrow\left(X_{2}, \tau_{2}\right)$ is a morphism in Spec then $\hat{\mathrm{D}}(g): \hat{\mathrm{D}}\left(X_{2}\right) \rightarrow \hat{\mathrm{D}}\left(X_{1}\right)$ given by $\hat{\mathrm{D}}(g)(U)=g^{-1}(U)$ is a morphism in BDL. If $A \in \mathrm{BDL}$ we write $\hat{\varphi}: A \rightarrow \hat{\mathrm{D}}(\hat{\mathrm{X}}(A))$ for the map given by $\hat{\varphi}(a)=\varphi(a)$ for every $a \in A$, which is an isomorphism in BDL. If $(X, \tau) \in$ Spec we write $\hat{\epsilon}:(X, \tau) \rightarrow \hat{\mathrm{X}}(\hat{\mathrm{D}}(X))$ for the map given by $\hat{\epsilon}(x)=\{U \in \mathrm{KO}(X, \tau): x \in U\}$, which is an isomorphism in Spec.

${ }^{4}$ Every sober topological space is automatically $T_{0}$. 
Proposition 2.9. The contravariant functors $\hat{\mathrm{X}}: \mathrm{BDL} \rightarrow \mathrm{Spec}$ and $\hat{\mathrm{D}}: \mathrm{Spec} \rightarrow$ $\mathrm{BDL}$ define a dual categorical equivalence with natural isomorphisms $\hat{\varphi}$ and $\hat{\epsilon}$.

It follows from propositions 2.8 and 2.9 that there is a dual equivalence between the categories PS and Spec. In what follows we give some definition in order to recall the known fact that the categories PS and Spec are isomorphic.

Let $(X, \tau)$ be a topological space. For every $U \subseteq X$ we write $\bar{U}$ to indicate the closure of $U$. The specialization order $\leq_{\tau}$ is defined by

$$
x \leq_{\tau} y \text { if and only if } x \in \overline{\{y\}} .
$$

The relation $\leq_{\tau}$ is a preorder and it is an order if $(X, \tau)$ is $T_{0}$. If $(X, \tau)$ is a spectral space then $\left(X, \leq_{\tau}, \tau^{*}\right)$ is a Priestley space, where $\tau^{*}$ is the topology with subbase $\left\{U \subseteq X: U \in \operatorname{KO}(X, \tau)\right.$ or $\left.U^{c} \in \operatorname{KO}(X, \tau)\right\}$. Moreover, $U$ is compact open in $(X, \tau)$ if and only if $U$ is a clopen upset in $\left(X, \leq_{\tau}, \tau^{*}\right)$. If $(X, \leq, \tau)$ is a Priestley space then $\left(X, \tau_{s}\right)$ is a spectral space, where $\tau_{s}$ is the set of open upsets of $(X, \leq, \tau)$. Moreover, $U$ is clopen upset in $(X, \leq, \tau)$ if and only if $U$ is compact open in $\left(X, \tau_{s}\right)$.

Proposition 2.10. The categories PS and Spec are isomorphic.

The following convention will be used throughout the paper.

Remark 2.11. If $(X, \leq, \tau)$ is a Priestley space we have defined $\mathrm{D}(X)$ as the set of clopen upsets of $(X, \leq, \tau)$. Let $(X, \tau)$ be a spectral space. We also write $\mathrm{D}^{*}(X)$ for the set of clopen upsets of its associated Priestley space $\left(X, \leq_{\tau}, \tau^{*}\right)$.

We also write SRL for the algebraic category of subresiduated lattices. Finally we recall the dual equivalence for SRL given in [4], which is based in Priestley duality.

A WH-space is a structure $(X, \leq, \tau, S)$ such that $(X, \leq, \tau)$ is a Priestley space, $(X, \leq, S)$ is a WH-frame, $S(x)$ is a closed set of $X$ for every $x \in X$ and $S^{-1}(Y)$ is clopen for every clopen subset $Y$. In [4, Proposition 4.6] can be found another characterization for WH-spaces. We say that a structure $(X, \leq, \tau, S)$ is a $\mathrm{pWH}$-space if it is a WH-space and $S$ a preorder. A function $f:\left(X_{1}, \leq_{1}, \tau_{1}, S_{1}\right) \rightarrow\left(X_{2}, \leq_{2}, \tau_{2}, S_{2}\right)$ between $\mathrm{pWH}$-spaces is called pWH-morphism if it is an order preserving continuous map such that: 1) for every $x, y \in X_{1}$, if $(x, y) \in S_{1}$ then $(f(x), f(y)) \in S_{2}$; 2) for every $x \in X_{1}$ and $z \in X_{2}$, if $(f(x), z) \in S_{2}$ then there is $y \in X_{1}$ such that $(x, y) \in S_{1}$ and $\mathrm{f}(\mathrm{y})=\mathrm{z}$. We write SRLS for the category whose objects are pWH-spaces and whose morphisms are pWH-morphisms.

Let $A \in \mathrm{SRL}$. We also write $\mathrm{X}(A)$ for $\left(X(A), \subseteq, \tau_{A}, S_{A}\right)$, which is an object of SRLS. Let $f: A_{1} \rightarrow A_{2}$ be a morphism in SRL. We also write $\mathrm{X}(f)$ for the map from $\mathrm{X}\left(A_{2}\right)$ to $\mathrm{X}\left(A_{1}\right)$ given by $\mathrm{X}(f)(P)=f^{-1}(P)$, which is a morphism in SRLS. Given $(X, \leq, \tau, S) \in \mathrm{SRLS}$ we also write $\mathrm{D}(X)$ for $\langle D(X), \cap, \cup, \Rightarrow, \emptyset, X\rangle$, which is in SRL. Finally, if $f:\left(X_{1}, \leq_{1}, \tau_{1}, S_{1}\right) \rightarrow\left(X_{2}, \leq_{2}, \tau_{2}, S_{2}\right)$ is a morphism in SRLS we also write $\mathrm{D}(f)$ for the map from $\mathrm{D}\left(X_{2}\right)$ to $\mathrm{D}\left(X_{1}\right)$ given by $\mathrm{D}(f)(U)=f^{-1}(U)$, which is a morphism in SRL. If $A \in \mathrm{SRL}$ we also write $\varphi$ for the map $\varphi: A \rightarrow \mathrm{D}(\mathrm{X}(A))$ defined as in (2.1).

For the following result see [4, Theorem 4.15] and [4, Subsection 4.1].

Theorem 2.12. The contravariant functors $\mathrm{X}: \mathrm{SRL} \rightarrow \mathrm{SRLS}$ and $\mathrm{D}: \mathrm{SRLS} \rightarrow \mathrm{SRL}$ defines a dual categorical equivalence with natural isomorphisms $\varphi$ and $\epsilon$.

\section{A speCtral Style DUALity For SUbresiduated lattices}

In this section we obtain a spectral style duality in the framework of SRL. 
We start with the following definition.

Definition 3.1. A p-spectral space is structure $(X, \tau, S)$ which satisfy the following conditions:

(1) $(X, \tau)$ is a spectral space.

(2) $S$ is a preorder on $X$.

(3) $S^{-1}\left(U \cap V^{c}\right)^{c} \in \mathrm{KO}(X, \tau)$ for every $U, V \in \mathrm{KO}(X, \tau)$.

(4) $S(x)=\bigcap\{U \in \mathrm{KO}(X, \tau): S(x) \subseteq U\}$ for every $x \in X$.

A p-spectral map is a function $f:\left(X_{1}, \tau_{1}, S_{1}\right) \rightarrow\left(X_{2}, \tau_{2}, S_{2}\right)$ between p-spectral spaces which satisfy the following conditions:

(1) $f:\left(X_{1}, \tau_{1}\right) \rightarrow\left(X_{2}, \tau_{2}\right)$ is a spectral function.

(2) For every $x, y \in X_{1}$, if $(x, y) \in S_{1}$ then $(f(x), f(y)) \in S_{2}$.

(3) For every $x \in X_{1}$ and $z \in X_{2}$, if $(f(x), z) \in S_{2}$ then there exists $y \in X_{1}$ such that $(x, y) \in S_{1}$ and $f(y)=z$.

We write SSpec for the category whose objects are p-spectral spaces and whose morphisms are p-spectral maps.

In particular, if $(X, \tau, S) \in \operatorname{SSpec}$ then $X \in \mathrm{KO}(X, \tau)$, so $X \Rightarrow_{S} U \in \operatorname{KO}(X, \tau)$ for every $U \in \operatorname{KO}(X, \tau)$.

If $f: X \rightarrow Y$ is a function and $U \subseteq X$, we define

$$
f[U]=\{f(u): u \in U\} .
$$

The conditions 2) and 3) of the definition of morphism in SSpec are equivalent to the following one: for every $x \in X_{1}, f\left[S_{1}(x)\right]=S_{2}(f(x))$.

Let $A \in \mathrm{SRL}$. We also use the notation $\hat{\mathrm{X}}(A)$ for $\left(X(A), \hat{\tau}_{A}, S_{A}\right)$.

Lemma 3.2. Let $A \in \mathrm{SRL}$. Then $\hat{\mathrm{X}}(A) \in \mathrm{SSpec}$.

Proof. Let $A \in \mathrm{SRL}$. It follows from Proposition 2.9 that $\left(X(A), \hat{\tau}_{A}\right)$ is a spectral space and it follows from Lemma 2.6 that $S_{A}$ is a preorder. Let $U, V \in$ $\mathrm{KO}\left(X(A), \hat{\tau}_{A}\right)$. Then there exist $a, b \in A$ such that $U=\varphi(a)$ and $V=\varphi(b)$. It follows from Theorem 2.3 that $U \Rightarrow_{S_{A}} V=\varphi(a \rightarrow b) \in \operatorname{KO}\left(X(A), \hat{\tau}_{A}\right)$. Finally we will see that

$$
S_{A}(P)=\bigcap\left\{\varphi(a): S_{A}(P) \subseteq \varphi(a)\right\} .
$$

for every $P \in X(A)$. Let $P \in X(A)$. Let $Q \in \bigcap\left\{\varphi(a): S_{A}(P) \subseteq \varphi(a)\right\}$. Suppose that $Q \notin S_{A}(P)$, so there exist $a, b \in A$ such that $a \rightarrow b \in P, a \in Q$ and $b \notin Q$. Notice that since $1 \rightarrow(a \rightarrow b)=a \rightarrow b$ and $a \rightarrow b \in P$ then $S_{A}(P) \subseteq \varphi(a \rightarrow b)$. In particular, $a \rightarrow b \in Q$. Since $Q$ is a filter then $a \wedge(a \rightarrow b) \in Q$. By using that $a \wedge(a \rightarrow b) \leq b$ and the fact that $Q$ is an upset we deduce that $b \in Q$, which is a contradiction. Hence, $\bigcap\left\{\varphi(a): S_{A}(P) \subseteq \varphi(a)\right\} \subseteq S_{A}(P)$. The converse inclusion is immediate.

Let $f: A_{1} \rightarrow A_{2}$ be a morphism in SRL. We also use the notation $\hat{\mathrm{X}}(f)$ : $\hat{\mathrm{X}}\left(A_{2}\right) \rightarrow \hat{\mathrm{X}}\left(A_{1}\right)$ for the map given by $\hat{\mathrm{X}}(f)(P)=f^{-1}(P)$.

Lemma 3.3. Let $f: A_{1} \rightarrow A_{2}$ be a morphism in $\mathrm{SRL}$. Then $\hat{\mathrm{X}}(f): \hat{\mathrm{X}}\left(A_{2}\right) \rightarrow \hat{\mathrm{X}}\left(A_{1}\right)$ is a morphism in SSpec.

Proof. Let $f: A_{1} \rightarrow A_{2}$ be a morphism in SRL. We know that $\hat{\mathrm{X}}(f)$ is a spectral function. The rest of the proof follows from [4, Theorem 4.14]. 
Thus, we have defined a contravariant functor

$$
\hat{\mathrm{X}}: \mathrm{SRL} \rightarrow \text { SSpec. }
$$

Remark 3.4. Let $(X, \tau)$ be a $T_{0}$ topological space. Hence, $\left(X, \leq_{\tau}\right)$ is a poset. Since every open set is an upset then $\mathrm{KO}(X, \tau) \subseteq \mathrm{Up}(X)$. Let $S$ be a binary relation on $X$ such that $\left(X, \leq_{\tau}, S\right)$ is a WH-frame. Also assume that $\mathrm{KO}(X, \tau)$ is closed under $\cap, \cup, \Rightarrow_{S}$ and $X$. Then $\operatorname{KO}(X, \tau)$ and $\mathrm{Up}(X)$ can be seen as algebras in the language $\left\{\cap, \cup, \Rightarrow_{S}, \emptyset, X\right\}$. Moreover, $\operatorname{KO}(X, \tau)$ is a subalgebra of $\operatorname{Up}(X)$ in this sense.

If $(X, \tau, S) \in$ SSpec we also use the notation $\hat{\mathrm{D}}(X)$ for the bounded distributive lattice $\operatorname{KO}(X, \tau)$ endowed with the binary operation $\Rightarrow_{S}$.

Lemma 3.5. Let $(X, \tau, S) \in$ SSpec. Then $\hat{\mathrm{D}}(X) \in \mathrm{SRL}$.

Proof. Let $(X, \tau, S) \in$ SSpec. Note that by Remark 3.4 and Proposition 2.5 we only need to show that $\left(X, \leq_{\tau}, S\right)$ is a WH frame. Let $(x, y) \in \leq_{\tau} \circ S$, so there exists $z \in X$ such that $x \leq_{\tau} z$ and $(z, y) \in S$. Suppose that $(x, y) \notin S$, so there exists $U \in \operatorname{KO}(X, \tau)$ such that $y \notin U$ and $S(x) \subseteq U$. Since $y \in S(z)$ and $y \notin U$ then $S(z) \nsubseteq U$, i.e., $z \in\left(X \Rightarrow_{S} U\right)^{c}$, which is a closed set. Since $x \in \overline{\{z\}}$ then $x \in\left(X \Rightarrow_{S} U\right)^{c}$. Thus, $S(x) \nsubseteq U$, which is a contradiction.

Let $g:\left(X_{1}, \tau_{1}, S_{1}\right) \rightarrow\left(X_{2}, \tau_{2}, S_{2}\right)$ be a morphism in SSpec. We also use the notation $\hat{\mathrm{D}}(g): \hat{\mathrm{D}}\left(X_{2}\right) \rightarrow \hat{\mathrm{D}}\left(X_{1}\right)$ for the map given by $\hat{\mathrm{D}}(g)(U)=g^{-1}(U)$.

Lemma 3.6. Let $g:\left(X_{1}, \tau_{1}, S_{1}\right) \rightarrow\left(X_{2}, \tau_{2}, S_{2}\right)$ be a morphism in SSpec. Then $\hat{\mathrm{D}}(g): \mathrm{KO}\left(X_{2}, \tau_{2}\right) \rightarrow \mathrm{KO}\left(X_{1}, \tau_{1}\right)$ is a morphism in $\mathrm{SRL}$.

Proof. Let $g:\left(X_{1}, \tau_{1}, S_{1}\right) \rightarrow\left(X_{2}, \tau_{2}, S_{2}\right)$ be a morphism in SSpec. In particular, $\hat{\mathrm{D}}(g)$ is a morphism of bounded distributive lattices. Since $g$ is a morphism in SSpec then $S_{2}(g(x))=g\left[S_{1}(x)\right]$, so a direct computation shows that $g^{-1}\left(U \Rightarrow_{S_{2}} V\right)=$ $g^{-1}(U) \Rightarrow_{S_{1}} g^{-1}(V)$ for every $U, V \in \mathrm{KO}\left(X_{2}, \tau_{2}\right)$. Therefore $\hat{\mathrm{D}}(g)$ is a morphism in $\mathrm{SRL}$.

Thus, we have defined a contravariant functor

$$
\hat{\mathrm{D}}: \mathrm{SSpec} \rightarrow \mathrm{SRL} \text {. }
$$

If $(X, \tau, S) \in \mathrm{SSpec}$ we also write $\hat{\epsilon}$ for the map $\hat{\epsilon}:(X, \tau, S) \rightarrow \hat{\mathrm{X}}(\hat{\mathrm{D}}(X))$ given by $\hat{\epsilon}(x)=\{U \in \operatorname{KO}(X, \tau): x \in U\}$.

Lemma 3.7. Let $(X, \tau, S) \in \mathrm{SSpec}$. Then $\hat{\epsilon}:(X, \tau, S) \rightarrow \hat{\mathrm{X}}(\hat{\mathrm{D}}(X))$ is an isomorphism in SSpec.

Proof. It is enough to prove that for every $x, y \in X,(x, y) \in S$ if and only if $(\hat{\epsilon}(x), \hat{\epsilon}(y)) \in S_{\hat{\mathrm{D}}(X)}$. Let $x, y \in X$. Suppose that $(x, y) \in S$. Let $U, V \in \operatorname{KO}(X, \tau)$ such that $U \Rightarrow_{S} V \in \hat{\epsilon}(x)$ and $U \in \hat{\epsilon}(y)$, i.e., $S(x) \cap U \subseteq V$ and $y \in U$. Taking into account that $y \in S(x) \cap U$ we obtain that $y \in V$. Thus, $(\hat{\epsilon}(x), \hat{\epsilon}(y)) \in S_{\hat{\mathrm{D}}(X)}$. Conversely, suppose that $(x, y) \notin S$, so there exists $U \in \operatorname{KO}(X, \tau)$ such that $y \notin$ $U$ and $S(x) \subseteq U$. Hence, $X \Rightarrow_{S} U \in \hat{\epsilon}(x), X \in \hat{\epsilon}(y)$ and $U \notin \hat{\epsilon}(y)$. Hence, $(\hat{\epsilon}(x), \hat{\epsilon}(y)) \notin S_{\hat{\mathrm{D}}(X)}$.

Let $A \in \mathrm{SRL}$. We also write $\hat{\varphi}: A \rightarrow \hat{\mathrm{D}}(\hat{\mathrm{X}}(A))$ for the map given by $\hat{\varphi}(a)=\varphi(a)$ for every $a \in A$, It follows from Theorem 2.3 that $\hat{\varphi}$ is an isomorphism in SRL.

The following theorem follows from Proposition 2.9 and the results of this section. 
Theorem 3.8. The contravariant functors $\hat{\mathrm{X}}: \mathrm{SRL} \rightarrow \mathrm{SSpec}$ and $\hat{\mathrm{D}}: \mathrm{SSpec} \rightarrow \mathrm{SRL}$ defines a dual categorical equivalence with natural isomorphisms $\hat{\varphi}$ and $\hat{\epsilon}$.

\section{A Bitopological Style DUALITy FOR SUBREsiduated LATtices}

A bitopological space is a structure $\left(X, \tau, \tau^{\prime}\right)$ where $(X, \tau)$ and $\left(X, \tau^{\prime}\right)$ are topological spaces. Inspired by the definition of subresiduated and the results explored in [2], in this section we introduce a category whose objects are certain bitopological spaces and we prove that this category is dually equivalent to SRL.

If $(X, \tau)$ is a topological space and $U \subseteq X$ we write $i n t_{\tau}(U)$ to indicate the interior of $U$.

Definition 4.1. A subresiduated bitopological space is a bitopological space $\left(X, \tau, \tau^{\prime}\right)$ which satisfy the following conditions:

(1) $(X, \tau)$ is a spectral space.

(2) $\left(X, \tau^{\prime}\right)$ is coherent.

(3) $\mathrm{KO}\left(X, \tau^{\prime}\right) \subseteq \mathrm{KO}(X, \tau)$.

(4) $\operatorname{int}_{\tau^{\prime}}\left(U^{c} \cup V\right) \in \mathrm{KO}(X, \tau)$ for every $U, V \in \mathrm{KO}(X, \tau)$.

A subresiduated bitopological morphism is a function $f:\left(X_{1}, \tau_{1}, \tau_{1}^{\prime}\right) \rightarrow\left(X_{2}, \tau_{2}, \tau_{2}^{\prime}\right)$ between SRL-spaces which satisfy the following conditions:

(1) $f:\left(X_{1}, \tau_{1}\right) \rightarrow\left(X_{2}, \tau_{2}\right)$ and $f:\left(X_{1}, \tau_{1}^{\prime}\right) \rightarrow\left(X_{2}, \tau_{2}^{\prime}\right)$ are spectral functions.

(2) $\operatorname{int}_{\tau_{1}^{\prime}}\left(f^{-1}\left(U^{c} \cup V\right)\right) \subseteq f^{-1}\left(\right.$ int $\left._{\tau_{2}^{\prime}}\left(U^{c} \cup V\right)\right)$ for every $U, V \in \mathrm{KO}\left(X_{2}, \tau_{2}\right)$.

We write BS for the category whose objects are subresiduated bitopological spaces and whose morphisms are subresiduated bitopological morphisms.

Note that if $\left(X, \tau, \tau^{\prime}\right) \in \mathrm{BS}$ then $\tau^{\prime} \subseteq \tau$ and

$$
\begin{aligned}
\mathrm{KO}\left(X, \tau^{\prime}\right) & =\mathrm{KO}(X, \tau) \cap \tau^{\prime} \\
& =\left\{U \in \operatorname{KO}(X, \tau): i n t_{\tau^{\prime}}(U)=U\right\} .
\end{aligned}
$$

Also notice that if $f:\left(X_{1}, \tau_{1}, \tau_{1}^{\prime}\right) \rightarrow\left(X_{2}, \tau_{2}, \tau_{2}^{\prime}\right)$ is a morphism in BS then $f:\left(X_{1}, \tau_{1}\right) \rightarrow$ $\left(X_{2}, \tau_{2}\right)$ and $f:\left(X_{1}, \tau_{1}^{\prime}\right) \rightarrow\left(X_{2}, \tau_{2}^{\prime}\right)$ are continuous maps.

Remark 4.2. Let $f:\left(X_{1}, \tau_{1}, \tau_{1}^{\prime}\right) \rightarrow\left(X_{2}, \tau_{2}, \tau_{2}^{\prime}\right)$ be a morphism in BS. Then

$$
\operatorname{int}_{\tau_{1}^{\prime}}\left(f^{-1}\left(U^{c} \cup V\right)\right)=f^{-1}\left(\text { int }_{\tau_{2}^{\prime}}\left(U^{c} \cup V\right)\right)
$$

for every $U, V \in \operatorname{KO}\left(X_{2}, \tau_{2}\right)$. We only need to show that $f^{-1}\left(\right.$ int $\left._{\tau_{2}^{\prime}}\left(U^{c} \cup V\right)\right) \subseteq$ int $_{\tau_{1}^{\prime}}\left(f^{-1}\left(U^{c} \cup V\right)\right)$ for every $U, V \in \operatorname{KO}\left(X_{2}, \tau_{2}\right)$. In order to show it, let $U, V \in$ $\mathrm{KO}\left(X_{2}, \tau_{2}\right)$. Since int $\tau_{2}^{\prime}\left(U^{c} \cup V\right) \subseteq U^{c} \cup V$ then $f^{-1}\left(\right.$ int $\left._{\tau_{2}^{\prime}}\left(U^{c} \cup V\right)\right) \subseteq f^{-1}\left(U^{c} \cup V\right)$. Taking into account that $f:\left(X_{1}, \tau_{1}^{\prime}\right) \rightarrow\left(X_{2} . \tau_{2}^{\prime}\right)$ is a spectral function we deduce that $f^{-1}\left(\right.$ int $\left._{\tau_{2}^{\prime}}\left(U^{c} \cup V\right)\right) \in \tau_{1}^{\prime}$, so $f^{-1}\left(\right.$ int $\left._{\tau_{2}^{\prime}}\left(U^{c} \cup V\right)\right) \subseteq$ int $_{\tau_{1}^{\prime}}\left(f^{-1}\left(U^{c} \cup V\right)\right)$.

Let $A \in \mathrm{SRL}$. Recall that $\hat{\tau}_{A}$ was defined as the topology on $X(A)$ generated by the base $\{\varphi(a): a \in A\}$. Moreover, since $D=\{a \in A: a=1 \rightarrow a\}$ is a bounded sublattice of $A$ then it is immediate that $\{\varphi(a): a \in D\}$ is also a base for a topology on $X(A)$, which will be denoted by $\bar{\tau}_{A}$. Inspired by the fact that $\operatorname{KO}\left(X(A), \hat{\tau}_{A}\right)=\{\varphi(a): a \in A\}$, in the following lemma we will show that $\mathrm{KO}\left(X(A), \bar{\tau}_{A}\right)=\{\varphi(a): a \in D\}$.

Lemma 4.3. Let $A \in \mathrm{SRL}$. Then $\operatorname{KO}\left(X(A), \bar{\tau}_{A}\right)=\{\varphi(a): a \in D\}$. 
Proof. Let $A \in \mathrm{SRL}$. Let $U \in \operatorname{KO}\left(X(A), \bar{\tau}_{A}\right)$, so there exist $a_{1}, \ldots, a_{n} \in D$ such that $U=\varphi\left(a_{1}\right) \cup \cdots \cup \varphi\left(a_{n}\right)$. Hence, $U=\varphi(a)$ with $a=a_{1} \vee \cdots \vee a_{n}$. Since $D$ is a bounded sublattice of $A$ then $a \in D$. Conversely, let $U \in \operatorname{KO}\left(X(A), \bar{\tau}_{A}\right)$. Then $U=\varphi(a)$ for some $a \in D$. In particular, $\varphi(a) \in \bar{\tau}_{A}$. Taking into account that $\varphi(a) \in \operatorname{KO}\left(X(A), \hat{\tau}_{A}\right)$ we conclude that $\varphi(a) \in \operatorname{KO}\left(X(A), \bar{\tau}_{A}\right)$.

It is immediate that if $A$ is a bounded distributive lattice and $a, b, c \in A$, then $a \wedge c \leq b$ if and only if $\varphi(c) \subseteq \varphi(a)^{c} \cup \varphi(b)$. This elemental remark will be used in the proof of the following lemma.

Lemma 4.4. Let $A \in \mathrm{SRL}$. Then $\left(X(A), \hat{\tau}_{A}, \bar{\tau}_{A}\right) \in \mathrm{BS}$.

Proof. Let $A \in \mathrm{SRL}$. In particular, $\left(X(A), \hat{\tau}_{A}\right)$ is a spectral space. It follows from Lemma 4.3 that $\left(X(A), \bar{\tau}_{A}\right)$ is coherent. Moreover, $\operatorname{KO}\left(X(A), \bar{\tau}_{A}\right) \subseteq \operatorname{KO}\left(X(A), \hat{\tau}_{A}\right)$ because $\{\varphi(a): a \in D\} \subseteq\{\varphi(a): a \in A\}$. Finally we will see that $i n t_{\tau^{\prime}}\left(U^{c} \cup V\right)$ belongs to $\mathrm{KO}(X, \tau)$ for every $U, V \in \mathrm{KO}\left(X(A), \hat{\tau}_{A}\right)$, where $\tau^{\prime}=\bar{\tau}_{A}$. Let $U, V \in$ $\operatorname{KO}\left(X(A), \hat{\tau}_{A}\right)$. Then there exist $a, b \in A$ such that $U=\varphi(a)$ and $V=\varphi(b)$. Moreover, $a \rightarrow b \in D$. We will see that

$$
\operatorname{int}_{\tau^{\prime}}\left(\varphi(a)^{c} \cup \varphi(b)\right)=\varphi(a \rightarrow b) .
$$

Let $P \in \operatorname{int}_{\tau^{\prime}}\left(\varphi(a)^{c} \cup \varphi(b)\right)$. Then there exists $c \in D$ such that $c \in P$ and $\varphi(c) \subseteq \varphi(a)^{c} \cup \varphi(b)$, so $a \wedge c \leq b$. Since $a \rightarrow(a \wedge c)=a \rightarrow c$ then $a \rightarrow c \leq a \rightarrow b$. But $c=1 \rightarrow c \leq a \rightarrow c$, so $c \leq a \rightarrow b$. Thus, $a \rightarrow b \in P$ because $c \in P$. Conversely, let $P \in \varphi(a \rightarrow b)$, so $a \rightarrow b \in P$. Since $a \wedge(a \rightarrow b) \leq b$ we conclude that $P \in \varphi(a \rightarrow b) \subseteq \varphi(a)^{c} \cup \varphi(b)$. Hence, $P \in$ int $_{\tau^{\prime}}\left(\varphi(a)^{c} \cup \varphi(b)\right)$. Therefore, int $_{\tau^{\prime}}\left(\varphi(a)^{c} \cup \varphi(b)\right)=\varphi(a \rightarrow b)$.

Let $A \in \mathrm{SRL}$. We define $\mathrm{F}(A)=\left(X(A), \hat{\tau}_{A}, \bar{\tau}_{A}\right)$. Let $f: A_{1} \rightarrow A_{2}$ be a morphism in SRL. We define $\mathrm{F}(f): \mathrm{F}\left(A_{2}\right) \rightarrow \mathrm{F}\left(A_{1}\right)$ by $\mathrm{F}(f)(P)=f^{-1}(P)$. In particular,

$$
\mathrm{F}(f)^{-1}(\varphi(a))=\varphi(f(a))
$$

for every $a \in A_{1}$. We shall use this fact in the proof of the following lemma.

Lemma 4.5. Let $f: A_{1} \rightarrow A_{2}$ be a morphism in SRL. Then $\mathrm{F}(f): \mathrm{F}\left(A_{2}\right) \rightarrow \mathrm{F}\left(A_{1}\right)$ is a morphism in $\mathrm{BS}$.

Proof. Let $f: A_{1} \rightarrow A_{2}$ be a morphism in SRL. In particular, $\mathrm{F}(f):\left(X\left(A_{2}\right), \tau_{\hat{A}_{2}}\right) \rightarrow$ $\left(X\left(A_{1}\right), \tau_{\hat{A}_{1}}\right)$ is a spectral function. Let $a \in A_{1}$ such that $1 \rightarrow a=a$, so $f(a) \in A_{2}$ and $1 \rightarrow f(a)=f(a)$. Besides, $\mathrm{F}(f)^{-1}(\varphi(a))=\varphi(f(a))$. Hence, it follows from Lemma 4.3 that $\mathrm{F}(f):\left(X\left(A_{2}\right), \bar{\tau}_{A_{2}}\right) \rightarrow\left(X\left(A_{1}\right), \bar{\tau}_{A_{1}}\right)$ is a spectral function too. Finally, let $U, V \in \operatorname{KO}\left(X\left(A_{1}\right), \tau_{A_{1}}\right)$. Then there exist $a, b \in A_{1}$ such that $U=\varphi(a)$ and $V=\varphi(b)$. Then it follows from the proof of Lemma 4.4 that

$$
\begin{gathered}
\operatorname{int}_{\bar{\tau}_{A_{2}}}\left(\mathrm{~F}(f)^{-1}\left(\varphi(a)^{c} \cup \varphi(b)\right)\right)=\varphi(f(a \rightarrow b)), \\
\mathrm{F}(f)^{-1}\left(\operatorname{int}_{\bar{\tau}_{A_{1}}}\left(\varphi(a)^{c} \cup \varphi(b)\right)\right)=\mathrm{F}(f)^{-1}(\varphi(a \rightarrow b)) .
\end{gathered}
$$

Thus,

$$
\operatorname{int}_{\bar{\tau}_{A_{2}}}\left(\mathrm{~F}(f)^{-1}\left(\varphi(a)^{c} \cup \varphi(b)\right)\right)=\mathrm{F}(f)^{-1}\left(\operatorname{int}_{\bar{\tau}_{A_{1}}}\left(\varphi(a)^{c} \cup \varphi(b)\right)\right) .
$$

Therefore, $\mathrm{F}(f)$ is a morphism in BS. 
The assignment $A \mapsto \mathrm{F}(A)$ and $f \mapsto \mathrm{F}(f)$ defines a contravariant functor

$$
\mathrm{F}: \mathrm{SRL} \rightarrow \mathrm{BS} \text {. }
$$

Let $\left(X, \tau, \tau^{\prime}\right)$ be a bitopological space. For every $U, V \in \operatorname{KO}(X, \tau)$ we define

$$
U \rightarrow \tau_{\tau^{\prime}} V=\operatorname{int}_{\tau^{\prime}}\left(U^{c} \cup V\right) \text {. }
$$

If there is not ambiguity or confusion we write $U \rightarrow V$ in place of $U \rightarrow_{\tau^{\prime}} V$. Let $\left(X, \tau, \tau^{\prime}\right) \in \mathrm{BS}$. Then, $\langle\operatorname{KO}(X, \tau), \cap, \cup, \emptyset, X\rangle$ is a bounded distributive lattice. Moreover, $\mathrm{KO}\left(X, \tau^{\prime}\right)=\{U \in \mathrm{KO}(X, \tau): X \rightarrow U=U\}$ and $\left\langle\mathrm{KO}\left(X, \tau^{\prime}\right), \cap, \cup, \emptyset, X\right\rangle$ is a bounded sublattice of $\langle\operatorname{KO}(X, \tau), \cap, \cup, \emptyset, X\rangle$. It follows from the definition of BS that $\langle\mathrm{KO}(X, \tau), \cap, \cup, \rightarrow, \emptyset, X\rangle$ is an algebra because $U \rightarrow V \in \operatorname{KO}(X, \tau)$ for every $U, V \in \mathrm{KO}(X, \tau)$. We define the following algebra of type $(2,2,2,0,0)$ : $\mathrm{G}(X)=\langle\mathrm{KO}(X, \tau), \cap, \cup, \rightarrow, \emptyset, X\rangle$.

Lemma 4.6. Let $\left(X, \tau, \tau^{\prime}\right) \in \mathrm{BS}$. Then $\mathrm{G}(X) \in \mathrm{SRL}$.

Proof. Let $\left(X, \tau, \tau^{\prime}\right) \in \mathrm{BS}$. Let $U, V$ and $W$ elements of $\operatorname{KO}(X, \tau)$. It is immediate that $U \rightarrow U=X$. Besides,

$$
\begin{aligned}
U \rightarrow(V \cap W) & =\operatorname{int}_{\tau^{\prime}}\left(U^{c} \cup(V \cap W)\right) \\
& =\operatorname{int}_{\tau^{\prime}}\left(U^{c} \cup V\right) \cap i n t_{\tau^{\prime}}\left(U^{c} \cup W\right) \\
& =(U \rightarrow V) \cap(U \rightarrow W)
\end{aligned}
$$

A similar argument shows that $(U \cup V) \rightarrow W=(U \rightarrow W) \cap(V \rightarrow W)$ and $(U \rightarrow V) \cap(V \rightarrow W) \subseteq U \rightarrow W$. In order to see that $U \cap(U \rightarrow V) \subseteq V$, let $x \in U \cap(U \rightarrow V)$. Then $x \in U$ and $x \in$ int $_{\tau^{\prime}}\left(U^{c} \cup V\right) \subseteq U^{c} \cup V$, so $x \in V$. Thus, $U \cap(U \rightarrow V) \subseteq V$. Finally, taking into account the inclusion int $t_{\tau^{\prime}}\left(U^{c} \cup V\right) \subseteq$ int $_{\tau^{\prime}}\left(W^{c} \cup \operatorname{int}_{\tau^{\prime}}\left(U^{c} \cup V\right)\right)$ we deduce $U \rightarrow V \subseteq W \rightarrow(U \rightarrow V)$.

Let $f:\left(X_{1}, \tau_{1}, \tau_{1}^{\prime}\right) \rightarrow\left(X_{2}, \tau_{2}, \tau_{2}^{\prime}\right)$ be a morphism in BS. We define $\mathrm{G}(f)$ : $\mathrm{G}\left(X_{2}\right) \rightarrow G\left(X_{1}\right)$ by $\mathrm{G}(f)(U)=f^{-1}(U)$. This map is a morphism of bounded distributive lattices. Moreover, it follows from Remark 4.2 that

$$
\mathrm{G}(f)(U \rightarrow V)=\mathrm{G}(f)(U) \rightarrow \mathrm{G}(f)(V)
$$

for every $U, V \in \operatorname{KO}\left(X, \tau_{2}\right)$. Therefore, we obtain the following lemma.

Lemma 4.7. Let $f:\left(X_{1}, \tau_{1}, \tau_{1}^{\prime}\right) \rightarrow\left(X_{2}, \tau_{2}, \tau_{2}^{\prime}\right)$ be a morphism in BS. Then $\mathrm{G}(f)$ : $\mathrm{G}\left(X_{2}\right) \rightarrow G\left(X_{1}\right)$ is a morphism in $\mathrm{SRL}$.

The assignment $\left(X, \tau, \tau^{\prime}\right) \mapsto \mathrm{G}(X)$ and $f \mapsto \mathrm{G}(f)$ defines a contravariant functor

$$
\mathrm{G}: \mathrm{BS} \rightarrow \mathrm{SRL} \text {. }
$$

Let $\left(X, \tau, \tau^{\prime}\right) \in \mathrm{BS}$. Then it follows from lemmas $4.3,4.4$ and 4.6 that

$$
\begin{aligned}
\mathrm{KO}\left(X(\mathrm{KO}(X, \tau)), \bar{\tau}_{\mathrm{KO}(X, \tau)}\right) & =\{\varphi(U): U \in \mathrm{KO}(X, \tau) \text { and } X \rightarrow U=U\} \\
& =\left\{\varphi(U): U \in \mathrm{KO}(X, \tau) \cap \tau^{\prime}\right\} \\
& =\left\{\varphi(U): U \in \mathrm{KO}\left(X, \tau^{\prime}\right)\right\} .
\end{aligned}
$$

Hence,

$$
\mathrm{KO}\left(X(\mathrm{KO}(X, \tau)), \bar{\tau}_{\mathrm{KO}(X, \tau)}\right)=\left\{\varphi(U): U \in \mathrm{KO}\left(X, \tau^{\prime}\right)\right\} .
$$

Our next goal is to show that if $\left(X, \tau, \tau^{\prime}\right) \in \mathrm{BS}$ then the map

$$
\tilde{\epsilon}:\left(X, \tau, \tau^{\prime}\right) \rightarrow\left(X(\mathrm{KO}(X, \tau)), \hat{\tau}_{\mathrm{KO}(X, \tau)}, \bar{\tau}_{\mathrm{KO}(X, \tau)}\right)
$$

give by $\widetilde{\epsilon}(x)=\{U \in \mathrm{KO}(X, \tau): x \in U\}$ is an isomorphism in BS. 
Proposition 4.8. Let $\left(X, \tau, \tau^{\prime}\right) \in \mathrm{BS}$. Then the map

$$
\tilde{\epsilon}:\left(X, \tau, \tau^{\prime}\right) \rightarrow\left(X(\mathrm{KO}(X, \tau)), \hat{\tau}_{\mathrm{KO}(X, \tau)}, \bar{\tau}_{\mathrm{KO}(X, \tau)}\right)
$$

is an isomorphism in $\mathrm{BS}$.

Proof. Let $\left(X, \tau, \tau^{\prime}\right) \in \mathrm{BS}$. In particular, $\widetilde{\epsilon}:(X, \tau) \rightarrow\left(X(\mathrm{KO}(X, \tau)), \hat{\tau}_{\mathrm{KO}(X, \tau)}\right)$ is an isomorphism of spectral spaces.

Consider now

$$
\tilde{\epsilon}:\left(X, \tau^{\prime}\right) \rightarrow\left(X(\mathrm{KO}(X, \tau)), \bar{\tau}_{\mathrm{KO}(X, \tau)}\right)
$$

Consider an element of $\operatorname{KO}\left(X(\operatorname{KO}(X, \tau)), \bar{\tau}_{\mathrm{KO}(X, \tau)}\right)$. It follows from (4.2) that this element takes the form $\varphi(U)$ for some $U \in \operatorname{KO}\left(X, \tau^{\prime}\right)$. Since $\widetilde{\epsilon}^{-1}(\varphi(U))=U$ we deduce that $\tilde{\epsilon}$ is a spectral function. On the other hand, consider the map

$$
\tilde{\epsilon}^{-1}:\left(X(\mathrm{KO}(X, \tau)), \bar{\tau}_{\mathrm{KO}(X, \tau)}\right) \rightarrow\left(X, \tau^{\prime}\right) .
$$

Let $U \in \operatorname{KO}\left(X, \tau^{\prime}\right)$. In particular, $\left(\widetilde{\epsilon}^{-1}\right)^{-1}(U)=\varphi(U)$. It follows from (4.2) that $\varphi(U) \in \operatorname{KO}\left(X(\mathrm{KO}(X, \tau)), \bar{\tau}_{\mathrm{KO}(X, \tau)}\right)$. Thus, $\widetilde{\epsilon}^{-1}$ is a spectral function too. Thus, $\widetilde{\epsilon}$ and $\widetilde{\epsilon}^{-1}$ are spectral functions.

Now consider the map

$$
\widetilde{\epsilon}:\left(X, \tau, \tau^{\prime}\right) \rightarrow\left(X(\mathrm{KO}(X, \tau)), \hat{\tau}_{\mathrm{KO}(X, \tau)}, \bar{\tau}_{\mathrm{KO}(X, \tau)}\right)
$$

and two arbitrary elements of $\mathrm{KO}\left(X(\mathrm{KO}(X, \tau)), \hat{\tau}_{\mathrm{KO}(X, \tau)}\right)$. These two elements have the form $\varphi(U)$ and $\varphi(V)$ for some $U, V \in \operatorname{KO}(X, \tau)$. We want to show that

$$
\widetilde{\epsilon}^{-1}(\varphi(U) \rightarrow \varphi(V))=\widetilde{\epsilon}^{-1}(\varphi(U)) \rightarrow \widetilde{\epsilon}^{-1}(\varphi(V)) .
$$

Since $\varphi$ is an isomorphism of subresiduated lattices we have that $\varphi(U) \rightarrow \varphi(V)=$ $\varphi(U \rightarrow V)$, so

$$
\begin{aligned}
\tilde{\epsilon}^{-1}(\varphi(U) \rightarrow \varphi(V)) & =\widetilde{\epsilon}^{-1}(\varphi(U \rightarrow V)) \\
& =U \rightarrow V \\
& =\widetilde{\epsilon}^{-1}(\varphi(U)) \rightarrow \widetilde{\epsilon}^{-1}(\varphi(V)) .
\end{aligned}
$$

Then $\tilde{\epsilon}$ is a morphism in BS. A similar argument show that $\widetilde{\epsilon}^{-1}$ is a morphism in BS. Hence, $\tilde{\epsilon}$ is an isomorphism in BS.

If $A \in \mathrm{BDL}$ we write $\widetilde{\varphi}$ for the map $\widetilde{\varphi}: A \rightarrow \mathrm{G}(\mathrm{F}(A))$ defined as $\widetilde{\varphi}=\varphi(a)$ for every $a \in A$.

Proposition 4.9. Let $A \in \mathrm{SRL}$. Then the map $\widetilde{\varphi}: A \rightarrow \mathrm{G}(\mathrm{F}(A))$ is an isomorphism in SRL.

Proof. Let $A \in \mathrm{SRL}$. Clearly, $\widetilde{\varphi}$ is morphism of bounded lattices. Let $\tau^{\prime}=\bar{\tau}_{A}$. It follows from the proof of Lemma 4.4 that for every $a, b \in A, \widetilde{\varphi}(a \rightarrow b)=$ $\operatorname{int}_{\tau^{\prime}}\left(\varphi(a)^{c} \cup \varphi(b)\right)$, i.e., $\widetilde{\varphi}(a \rightarrow b)=\widetilde{\varphi}(a) \rightarrow_{\tau^{\prime}} \widetilde{\varphi}(b)$. Therefore, $\widetilde{\varphi}$ is an isomorphism in SRL.

Therefore, we obtain the following result.

Theorem 4.10. The contravariant functors $\mathrm{F}: \mathrm{SRL} \rightarrow \mathrm{BS}$ and $\mathrm{G}: \mathrm{BS} \rightarrow \mathrm{SRL}$ define a dual categorical equivalence with natural isomorphisms $\widetilde{\varphi}$ and $\widetilde{\epsilon}$. 


\section{CAtegorical isomorphisms}

It follows from theorems 2.12, 3.8 and 4.10 that the categories SRLS, SSpec and BS are equivalent. In this last section we will show that in fact the categories SRLS, SSpec and BS are isomorphic.

Let $(X, \tau)$ be a coherent topological space and let $x, y \in X$. Notice that $x \leq_{\tau} y$ if and only if for every $U \in \mathrm{KO}(X, \tau)$, if $x \in U$ then $y \in U$. This elemental remark will be used throughout this section.

\subsection{Categorical isomorphism between SRLS and SSpec.}

Lemma 5.1. Let $(X, \tau, S) \in$ SSpec. Then $\left(X, \leq_{\tau}, \tau^{*}, S\right) \in$ SRLS.

Proof. Let $(X, \tau, S) \in$ SSpec. In particular, $\left(X, \leq_{\tau}, \tau^{*}\right)$ is a Priestley space and $S$ is a preorder. Now we will prove that $\left(X, \leq_{\tau}, S\right)$ is a WH-frame. Let $x \leq_{\tau} y$ and $(y, z) \in S$. We need to show that $z \in S(x)$. Suppose that $z \notin S(x)$. Then there exists $U \in \operatorname{KO}(X, \tau)$ such that $S(x) \subseteq U$ and $z \notin U$. Notice that since $S$ is reflexive and $x \leq_{\tau} y$ then $y \in \bigcap\{V \in \operatorname{KO}(X, \tau): S(x) \subseteq V\}$. Indeed, let $V \in \operatorname{KO}(X, \tau)$ such that $S(x) \subseteq V$. Then $x \in S(x) \subseteq V$, so $x \in V$. Taking into account that $x \leq_{\tau} y$ we obtain that $y \in V$. Thus,

$$
y \in \bigcap\{V \in \operatorname{KO}(X, \tau): S(x) \subseteq V\}=S(x) .
$$

Then $(x, y) \in S$ and $(y, z) \in S$, so it follows from the transitivity of $S$ that $z \in S(x)$. Then $z \in U$, which is a contradiction. We have proved that $\left(X, \leq_{\tau}, S\right)$ is a WHframe. Let $x \in X$. In order to prove that $S(x)$ is closed in $\left(X, \tau^{*}\right)$, let $y \notin S(x)$. Then there exists $U \in \mathrm{KO}(X, \tau)=\mathrm{D}^{*}(X)$ such that $y \notin U$ and $S(x) \subseteq U$, so $y \in U^{c} \subseteq S(x)^{c}$ and $U^{c}$ is an open set in $\left(X, \tau^{*}\right)$. Thus, $S(x)$ is closed in $\left(X, \tau^{*}\right)$ for every $x \in X$. Finally, let $W$ be a clopen in $\left(X, \tau^{*}\right)$, Then there exist $U, V \in$ $\mathrm{D}^{*}(X)=\mathrm{KO}(X, \tau)$ such that $W=U \cap V^{c}$. Then $S^{-1}(W)^{c}=S^{-1}\left(U \cap V^{c}\right)^{c} \in$ $\mathrm{KO}(X, \tau)=\mathrm{D}^{*}(X)$. Therefore, $S^{-1}(W)$ is clopen in $\left(X, \tau^{*}\right)$.

The following lemma is part of the folklore of Priestley spaces $[2,6]$.

Lemma 5.2. Let $(X, \leq, \tau)$ be a Priestley space. Let $C$ be a closed upset and $x \in X$ such that $x \notin C$. Then there exists $U \in \mathrm{D}(X)$ such that $C \subseteq U$ and $x \notin C$.

Now we show the following result.

Lemma 5.3. Let $(X, \leq, \tau, S) \in \mathrm{SRLS}$. Then $\left(X, \tau_{s}, S\right) \in \mathrm{SSpec}$.

Proof. Let $(X, \leq, \tau, S) \in \mathrm{SRLS}$. Then $\left(X, \tau_{s}\right)$ is a spectral space and $S$ is a preorder. Let $U, V \in \operatorname{KO}\left(X, \tau_{s}\right)=\mathrm{D}(X)$. Then $S^{-1}\left(U \cap V^{c}\right)^{c}$ is clopen in $(X, \tau)$. It follows from that $(X, \leq, S)$ is a WH-frame that $S(y) \subseteq S(x)$ whenever $x \leq y$, which implies that $S^{-1}\left(U \cap V^{c}\right)^{c}=\{x \in X: S(x) \cap U \subseteq V\}$ is an upset in $(X, \leq)$. Thus, $S^{-1}\left(U \cap V^{c}\right)^{c} \in \mathrm{D}(X)=\mathrm{KO}\left(X, \tau_{s}\right)$. Finally we will show that for every $x \in X$ we have that

$$
S(x)=\bigcap\left\{U \in \mathrm{KO}\left(X, \tau_{s}\right): S(x) \subseteq U\right\} .
$$

Let $x \in X$. The inclusion $S(x) \subseteq \bigcap\left\{U \in \mathrm{KO}\left(X, \tau_{s}\right): S(x) \subseteq U\right\}$ is immediate. Conversely, let $y \notin S(x)$. We know that $S(x)$ is closed in $(X, \tau)$. Besides $S(x)$ is an upset of $(X, \leq)$. Indeed, let $y, z \in X$ such that $y \in S(x)$ and $y \leq z$. Taking into account that $(X, \leq, S)$ is a WH-frame we have that $S(z) \subseteq S(y)$. Since $S$ is reflexive then $z \in S(z)$, so $z \in S(y)$. Thus, $(x, y) \in S$ and $(y, z) \in S$, so the transitivity of $S$ implies that $z \in S(x)$. Hence, $S(x)$ is a closed upset. Since $y \notin S(x)$ then it follows 
from Lemma 5.2 that there exists $U \in \mathrm{D}(X)$ such that $S(x) \subseteq U$ and $y \notin U$. Since $U \in \mathrm{KO}\left(X, \tau_{s}\right)$ we have that $y \notin \bigcap\left\{U \in \mathrm{KO}\left(X, \tau_{s}\right): S(x) \subseteq U\right\}$, which was our aim.

Therefore we obtain the following result.

Theorem 5.4. The categories SRLS and SSpec are isomorphic.

5.2. Categorical isomorphism between SRLS and BS. Let $X$ be a set, $U \subseteq X$ and $S \subseteq X \times X$. We say that $U$ is $S$-closed if $S(x) \subseteq U$ for every $x \in U$. Let $(X, \leq, \tau, S) \in$ SRLS. We write $\mathrm{D}_{\mathrm{S}}(X)$ for the elements of $\mathrm{D}(X)$ which are $S$-closed. We also define $\tau_{S}=\left\{U \in \tau_{s}: X \Rightarrow U=U\right\}$. It follows from the reflexivity of $S$ that $\tau_{S}=\left\{U \in \tau_{s}: U\right.$ is $S$-closed $\}$. Note that $\left(X, \tau_{S}\right)$ is a topological space.

Lemma 5.5. Let $(X, \leq, \tau, S) \in \mathrm{SRLS}$. Then the following conditions are satisfied:

(1) $U \Rightarrow V \in \mathrm{D}_{\mathrm{S}}(X)$ for every $U, V \in \mathrm{D}(X)$.

(2) If $(x, y) \notin S$ then there exists $U \in \mathrm{D}_{\mathrm{S}}(X)$ such that $x \in U$ and $y \notin U$.

(3) $\mathrm{D}_{\mathrm{S}}(X)$ is a base for a topology on $X$. Moreover, the topology generated by $\mathrm{D}_{\mathrm{S}}(X)$ is equal to $\tau_{S}$ and $\mathrm{D}_{\mathrm{S}}(X)=\mathrm{KO}\left(X, \tau_{S}\right)$.

Proof. In order to show 1) let $U, V \in \mathrm{D}(X)$. It follows from Theorem 2.12 that $U \Rightarrow V \in \mathrm{D}(X)$. The fact that $U \Rightarrow V$ is $S$-closed follows from the reflexivity and transitivity of $S$.

Now we shall see 2). Let $x, y \in X$ such that $(x, y) \notin S$. Since $S(x)$ is a closed set in a Priestley space then there exist $U, V \in \mathrm{D}(X)$ such that $S(x) \cap\left(U \cap V^{c}\right)=\emptyset$ and $y \in U \cap V^{c}$. Then $x \in U \Rightarrow V$ and $y \notin U \Rightarrow V$. Indeed, suppose that $y \in U \Rightarrow V$, i.e., $S(y) \cap U \subseteq V$. Since $y \in S(y) \cap U$ then $y \in V$, which is a contradiction. The fact that $U \Rightarrow V \in \mathrm{D}_{\mathrm{S}}(X)$ follows from 1$)$.

3) It follows from a straightforward computation that $\mathrm{D}_{\mathrm{S}}(X)$ is a base for a topology on $X$. In what follows we will prove that the topology generated by $\mathrm{D}_{\mathrm{S}}(X)$ is equal to $\tau_{S}$. It is immediate that every element of the topology generated by $\mathrm{D}_{\mathrm{S}}(X)$ belongs to $\tau_{S}$. Conversely, consider $U \in \tau_{S}$ and $x \in U$. In particular, $S(x) \subseteq U$. Let $y \notin U$, so $(x, y) \notin S$. Thus, by item 2$)$ there exists $W_{y} \in \mathrm{D}_{\mathrm{S}}(X)$ such that $x \in W_{y}$ and $y \notin W_{y}$. Hence, $U^{c} \subseteq \bigcup_{y \notin S(x)} W_{y}^{c}$. Since $U^{c}$ is closed in $(X, \tau)$ and $(X, \leq, \tau)$ is a Priestley space then $U^{c}$ is compact in $(X, \tau)$. Then there exist $W_{y_{1}}, \ldots, W_{y_{n}}$ such that $U^{c} \subseteq W_{y_{1}}^{c} \cup \cdots \cup W_{y_{n}}^{c}$. Hence, $x \in W_{y_{1}} \cap \cdots \cap W_{y_{n}} \subseteq U$. Taking into account that $W_{y_{1}} \cap \cdots \cap W_{y_{n}} \in \mathrm{D}_{\mathrm{S}}(X)$, we have that the topology generated by the base $\mathrm{D}_{\mathrm{S}}(X)$ is $\tau_{S}$.

Finally we will show that $\mathrm{D}_{\mathrm{S}}(X)=\mathrm{KO}\left(X, \tau_{S}\right)$. Let $U \in \mathrm{D}_{\mathrm{S}}(X)$, so $U \in \mathrm{D}(X)$ and $U$ is $S$-closed. In particular, $U \in \tau_{S}$. Also notice that $U$ is closed in $(X, \tau)$, so it is compact in $(X, \tau)$. Let $\left\{U_{i}\right\}_{i \in I} \subseteq \mathrm{D}_{\mathrm{S}}(X)$ such that $U \subseteq \bigcup_{i \in I} U_{i}$. Since $\{U\}_{i \in I} \subseteq \tau$ then there exist $U_{i_{1}}, \ldots, U_{i_{n}}$ such that $U \subseteq U_{i_{1}} \cup \cdots \cup U_{i_{n}}$. Thus, $U \in \mathrm{KO}\left(X, \tau_{S}\right)$. Conversely, let $U \in \mathrm{KO}\left(X, \tau_{S}\right)$. Then there exist $V_{1}, \ldots, V_{k} \in$ $\mathrm{D}_{\mathrm{S}}(X)$ such that $V=V_{1} \cup \cdots \cup V_{k}$, so $U \in \mathrm{D}(X)$. Moreover, $U$ is $S$-closed because $V_{1}, \ldots, V_{k}$ are $S$-closed. Then, $U \in \mathrm{D}_{\mathrm{S}}(X)$. Therefore, $\mathrm{D}_{\mathrm{S}}(X)=\mathrm{KO}\left(X, \tau_{S}\right)$.

Lemma 5.6. Let $(X, \leq, \tau, S) \in \mathrm{SRLS}$. Then $\left(X, \tau_{s}, \tau_{S}\right) \in \mathrm{BS}$.

Proof. Let $(X, \leq, \tau, S) \in \mathrm{SRLS}$. In particular, $\left(X, \tau_{s}\right)$ is a spectral space. It follows from Lemma 5.5 that $\left(X, \tau_{S}\right)$ is coherent. Let $U, V \in \operatorname{KO}\left(X, \tau_{s}\right)=\mathrm{D}(X)$. We have that $U \Rightarrow V=U \rightarrow V$, where $\rightarrow$ was defined in (4.1) of Section 4. Indeed, let $x \in U \Rightarrow V$. The reflexivity of $S$ shows that $U \Rightarrow V \subseteq U^{c} \cup V$. Then $x \in U^{c} \cup V$. 
Hence, $x \in U \rightarrow V$. Conversely, let $x \in U \rightarrow V$. Then there exists $W \in \mathrm{D}_{\mathrm{S}}(X)$ such that $x \in W \subseteq U^{c} \cup V$. By Lemma 5.5 we have that $U \Rightarrow V \in \mathrm{D}_{\mathrm{S}}(X)$, so $S(x) \subseteq W \subseteq U^{c} \cup V$, so $S(x) \cap U \subseteq V$. Thus, $x \in U \Rightarrow V$. We have proved that $U \Rightarrow V=\bar{U} \rightarrow V$. It follows from Theorem 2.12 that $U \Rightarrow V \in \operatorname{KO}\left(X, \tau_{s}\right)$, so $U \rightarrow V \in \mathrm{KO}\left(X, \tau_{s}\right)$. Therefore, $\left(X, \tau_{s}, \tau_{S}\right) \in \mathrm{BS}$.

Let $\left(X, \leq_{i}, \tau_{i}\right)$ be Priestley spaces for $i=1,2$. We write $\left(X,\left(\tau_{i}\right)_{s}\right)$ in place of $\left(X, \tau_{i s}\right)$ for $i=1,2$.

Lemma 5.7. Let $f:\left(X_{1}, \leq_{1}, \tau_{1}, S_{1}\right) \rightarrow\left(X_{2}, \leq_{2}, \tau_{2}, S_{2}\right)$ be a morphism in SRLS. Then $f:\left(X_{1},\left(\tau_{1}\right)_{s}, \tau_{S_{1}}\right) \rightarrow\left(X_{2},\left(\tau_{2}\right)_{s}, \tau_{S_{2}}\right)$ is a morphism in BS.

Proof. Let $f:\left(X_{1}, \leq_{1}, \tau_{1}, S_{1}\right) \rightarrow\left(X_{2}, \leq_{2}, \tau_{2}, S_{2}\right)$ be a morphism in SRLS. We know that $f:\left(X_{1},\left(\tau_{1}\right)_{s}\right) \rightarrow\left(X_{2},\left(\tau_{2}\right)_{s}\right)$ is a spectral function. Now consider $f$ : $\left(X_{1}, \tau_{S_{1}}\right) \rightarrow\left(X_{2}, \tau_{S_{2}}\right)$. Let $U \in \mathrm{KO}\left(X, \tau_{S_{2}}\right)=\mathrm{D}_{\mathrm{S}}\left(X_{2}\right)$. Since $U \in \mathrm{D}\left(X_{2}\right)$ and $f$ is a morphism of Priestley spaces then $f^{-1}(U) \in \mathrm{D}\left(X_{1}\right)$. A direct computation based in the facts that $U$ is $S_{2}$-closed and $(f(x), f(y)) \in S_{2}$ whenever $(x, y) \in S_{1}$ shows that $f^{-1}(U)$ is $S$-closed. Then $f^{-1}(U) \in \mathrm{D}_{\mathrm{S}}\left(X_{1}\right)=\operatorname{KO}\left(X_{1}, \tau_{S_{1}}\right)$. Thus, $f$ is also a spectral function. Finally, let $U, V \in \operatorname{KO}\left(X_{2},\left(\tau_{2}\right)_{s}\right)=\mathrm{D}\left(X_{2}\right)$. It follows from the proof of Lemma 5.6 that $U \rightarrow V=U \Rightarrow V$. It follows from Theorem 2.12 that $f^{-1}(U \Rightarrow V)=f^{-1}(U) \Rightarrow f^{-1}(V)$, so $f^{-1}(U \rightarrow V)=f^{-1}(U) \rightarrow f^{-1}(V)$. Therefore, $f:\left(X_{1},\left(\tau_{1}\right)_{s}, \tau_{S_{1}}\right) \rightarrow\left(X_{2},\left(\tau_{2}\right)_{s}, \tau_{S_{2}}\right)$ is a morphism in BS.

Straightforward computations show that we have a functor from SRLS to BS.

Lemma 5.8. Let $\left(X, \tau, \tau^{\prime}\right) \in$ BS. Then $\left(X, \leq_{\tau}, \tau^{*}, \leq_{\tau^{\prime}}\right) \in$ SRLS.

Proof. Let $\left(X, \tau, \tau^{\prime}\right) \in$ BS. Then $\left(X, \leq_{\tau}, \tau^{*}\right)$ is a Priestley space and $\leq_{\tau^{\prime}}$ is a preorder. It follows from that $\mathrm{KO}\left(X, \tau^{\prime}\right) \subseteq \mathrm{KO}(X, \tau)$ and $\left(X, \tau^{\prime}\right)$ is coherent that $\left(X, \leq_{\tau}, \leq_{\tau^{\prime}}\right)$ is a WH-frame. The fact that $\left(X, \tau^{\prime}\right)$ is coherent also shows that $\leq_{\tau^{\prime}}(x)$ is closed in $\left(X, \tau^{*}\right)$ for every $x \in X$. Now we will prove that for every $U, V \in \mathrm{KO}(X, \tau), U \Rightarrow V=U \rightarrow V$. Let $U, V \in \mathrm{KO}(X, \tau)$. Let $x \in U \Rightarrow V$. Then $\leq_{\tau^{\prime}}(x) \cap U \subseteq V$, so $\leq_{\tau^{\prime}}(x) \subseteq U^{c} \cup V$. Let $y \in U \cap V^{c}$, so $(x, y) \notin \leq_{\tau^{\prime}}$. Since $\operatorname{KO}\left(X, \tau^{\prime}\right)$ is coherent then there exists $W_{y} \in \mathrm{KO}\left(X, \tau^{\prime}\right)$ such that $x \in W_{y}$ and $y \notin W_{y}$. We have proved that $U \cap V^{c} \subseteq \bigcup_{(x, y) \notin \leq_{\tau^{\prime}}} W_{y}^{c}$. Since every $W_{y} \in$ $\mathrm{KO}\left(X, \tau^{\prime}\right) \subseteq \mathrm{KO}(X, \tau)=\mathrm{D}(X)$ and $U \cap V^{c}$ is closed in the Priestley space $\left(X, \tau^{*}\right)$ then there exist $W_{y_{1}}, \ldots, W_{y_{n}}$ such that $U \cap V^{c} \subseteq W_{y_{1}}^{c} \cup \cdots \cup W_{y_{n}}^{c}$. Thus, $W_{y_{1}}^{c} \cap$ $\cdots \cap W_{y_{n}}^{c} \in \tau^{\prime}$ and $x \in W_{y_{1}}^{c} \cap \cdots \cap W_{y_{n}}^{c} \subseteq U^{c} \cup V$, so $x \in U \rightarrow V$. Conversely, let $x \in U \rightarrow V$. Since $\left(X, \tau^{\prime}\right)$ is coherent we have that there exists $W \in \operatorname{KO}\left(X, \tau^{\prime}\right)$ such that $x \in W \subseteq U^{c} \cup V$. Let $y \in \leq_{\tau^{\prime}}(x) \cap U$, i.e., $x \leq_{\tau^{\prime}} y$ and $y \in U$. Since $x \in W$ then $y \in W$, so $y \in V$. Thus, $x \in U \Rightarrow V$. We have proved that for every $U, V \in \mathrm{KO}(X, \tau)$ we have that

$$
U \Rightarrow V=U \rightarrow V \text {. }
$$

Let $Z$ be a clopen in $(X, \tau)$, so there exist $U, V \in \mathrm{D}(X)$ such that $Z=U \cap V^{c}$. Moreover, a direct computation based in that $\leq_{\tau^{\prime}}$ is a preorder shows the equality $\left(\leq_{\tau^{\prime}}^{-1}(Z)\right)^{c}=U \Rightarrow V$. But by (5.1) we have that $U \Rightarrow V=U \rightarrow V$, so $\left(\leq_{\tau^{\prime}}^{-1}\right.$ $(Z))^{c}=U \rightarrow V \in \mathrm{KO}(X, \tau)$. Hence, $\leq_{\tau^{\prime}}^{-1}(Z)$ is a clopen in $\left(X, \tau^{*}\right)$.

Let $\left(X, \tau, \tau^{\prime}\right) \in$ BS. We have proved in Lemma 5.8 that $\left(X, \leq_{\tau}, \tau^{*}, \leq_{\tau^{\prime}}\right) \in$ SRLS. It is interesting to note that the relation $\leq_{\tau}$ is an order and that the relation $\leq_{\tau^{\prime}}$ is a preorder which is not necessarily an order. 
Remark 5.9. Let $\left(X, \tau, \tau^{\prime}\right) \in$ BS. By Lemma 5.8 we have that $\left(X, \leq_{\tau}, \tau^{*}, \leq_{\tau^{\prime}}\right) \in$ SRLS. Moreover, by the the proof of Lemma 5.8 we have that $X \Rightarrow U=X \rightarrow U$ for every $U \in \mathrm{KO}(X, \tau)$. Then

$$
\begin{aligned}
\mathrm{D}_{\mathrm{S}}(X) & =\{U \in \operatorname{KO}(X, \tau): X \Rightarrow U=U\} \\
& =\{U \in \operatorname{KO}(X, \tau): X \rightarrow U=U\} \\
& =\mathrm{KO}\left(X, \tau^{\prime}\right) .
\end{aligned}
$$

Therefore,

$$
\mathrm{D}_{\mathrm{S}}(X)=\mathrm{KO}\left(X, \tau^{\prime}\right)
$$

Lemma 5.10. Let $f:\left(X_{1}, \tau_{1}, \tau_{1}^{\prime}\right) \rightarrow\left(X_{2}, \tau_{2}, \tau_{2}^{\prime}\right)$ be a morphism in BS. Then $f:\left(X_{1}, \leq_{\tau_{1}}, \tau_{1}^{*}, \leq_{\tau_{1}^{\prime}}\right) \rightarrow\left(X_{2}, \leq_{\tau_{2}}, \tau_{2}^{*}, \leq_{\tau_{2}^{\prime}}\right)$ is a morphism in SRLS.

Proof. Let $f:\left(X_{1}, \tau_{1}, \tau_{1}^{\prime}\right) \rightarrow\left(X_{2}, \tau_{2}, \tau_{2}^{\prime}\right)$ be a morphism in BS. We know that $f:\left(X_{1}, \leq_{\tau_{1}}, \tau_{1}^{*}\right) \rightarrow\left(X_{2}, \leq_{\tau_{2}}, \tau_{2}^{*}\right)$ is a morphism of Piestley spaces. The fact that for every $x, y \in X_{1}$ it holds that $(f(x), f(y)) \in \leq_{\tau_{2}^{\prime}}$ whenever $(x, y) \in \leq_{\tau_{1}^{\prime}}$ follows from that $f:\left(X_{1}, \leq_{\tau_{1}^{\prime}}\right) \rightarrow\left(X_{2}, \leq_{\tau_{2}^{\prime}}\right)$ is a spectral function.

Let $S_{i}=\leq_{\tau_{i}^{\prime}}$ for $i=1,2$. Consider $x \in X_{1}$ and $z \in X_{2}$ such that $(f(x), z) \in S_{2}$. We need to show that there exists $y \in S_{1}(x)$ such that $f(y)=z$. Suppose that for every $y \in S_{1}(x)$ it holds that $f(y) \neq z$. Then there exist $U_{y}, V_{y} \in \mathrm{D}(X)$ such that $f(y) \in U_{y} \cap V_{y}^{c}$ and $z \notin U_{y} \cap V_{y}^{c}$. Thus,

$$
S_{1}(x) \subseteq \bigcup_{y \in S_{1}(x)} f^{-1}\left(U_{y} \cap V_{y}^{c}\right)
$$

Since $S_{1}(x)$ is closed in $\left(X, \tau^{*}\right)$ then there exist $U_{y_{1}}, \ldots, U_{y_{n}}, V_{y_{1}}, \ldots, V_{y_{n}}$ such that $S_{1}(x) \subseteq \bigcup_{i=1}^{n} f^{-1}\left(U_{y_{i}} \cap V_{y_{i}}^{c}\right)$, i.e.,

$$
\bigcap_{i=1}^{n} f^{-1}\left(U_{y_{i}}^{c} \cup V_{y_{i}}\right) \subseteq S_{1}(x)^{c} .
$$

A direct computation shows that $S_{1}(x)^{c}$ is open in $\left(X, \tau_{1}^{\prime}\right)$. Then by using that $f$ is a morphism in BS we obtain that

$$
\bigcap_{i=1}^{n} f^{-1}\left(i n t_{\tau_{2}^{\prime}}\left(U_{y_{i}}^{c} \cup V_{y_{i}}\right)\right) \subseteq S_{1}(x)^{c},
$$

which is equivalent to

$$
S_{1}(x) \subseteq \bigcup_{i=1}^{n}\left[f^{-1}\left(\operatorname{int}_{\tau_{2}^{\prime}}\left(U_{y_{i}}^{c} \cup V_{y_{i}}\right)\right]^{c} .\right.
$$

Since $x \in S_{1}(x)$ then there exists $j=1, \ldots, n$ such that $f(x) \notin i n t_{\tau_{2}^{\prime}}\left(U_{y_{j}}^{c} \cup V_{y_{j}}\right)$. Moreover, since $z \notin U_{y_{j}} \cap V_{y_{j}}^{c}$ then $z \notin i n t_{\tau_{2}^{\prime}}\left(U_{y_{j}} \cap V_{y_{j}}^{c}\right)$. It follows from $(f(x), z) \in S_{2}$ that $f(x) \notin i n t_{\tau_{2}^{\prime}}\left(U_{y_{j}} \cap V_{y_{j}}^{c}\right)$. Thus, $f(x) \in \emptyset$, which is a contradiction.

A direct computation shows that we have a functor from BS to SRLS.

Theorem 5.11. The categories SRLS and BS are isomorphic.

Proof. Let $(X, \leq, \tau, S) \in \mathrm{SRLS}$, so $\left(X, \tau_{s}, \tau_{S}\right) \in \mathrm{BS}$ and then $\left(X, \leq_{\tau_{s}}, \tau_{s}^{*}, \leq_{\tau_{S}}\right) \in$ SRLS. We know that $\leq=\leq_{\tau_{s}}$. Besides, for $x, y \in X$ it follows from Lemma 5.5 that $x \leq_{\tau_{S}} y$ if and only if for every $U \in \mathrm{D}_{\mathrm{S}}(X)$, if $x \in U$ then $y \in U$. Again by Lemma 5.5 the last condition is equivalent to saying that $(x, y) \in S$. Thus, $S=\leq_{\tau_{S}}$. 
Conversely, let $\left(X, \tau, \tau^{\prime}\right) \in \mathrm{BS}$. In consequence, $\left(X, \leq_{\tau}, \tau^{*}, \leq_{\tau^{\prime}}\right) \in \mathrm{SRLS}$ and then $\left(X, \tau_{s}^{*}, \tau_{\tau_{\tau^{\prime}}}^{*}\right) \in \mathrm{BS}$. We know that $\tau=\tau_{s}^{*}$. Define $S=\leq_{\tau^{\prime}}$. We will show that $\tau^{\prime}=\tau_{S}^{*}$. The fact that $\tau^{\prime} \subseteq \tau_{S}^{*}$ is immediate. Conversely, let $U \in \tau_{S}^{*}$. Let $x \in U$. Then it follows from Lemma 5.5 that there exists $V \in \mathrm{D}_{\mathrm{S}}(X)$ such that $x \in V \subseteq U$, so by Remark 5.9 we deduce that $V \in \mathrm{KO}\left(X, \tau^{\prime}\right)$. Then, $\tau_{S}^{*} \subseteq \tau^{\prime}$. Therefore, $\tau^{\prime}=\tau_{S}^{*}$.

\section{REFERENCES}

[1] Balbes R. and Dwinger P.:Distributive Lattices. University of Missouri Press (1974).

[2] Bezhanishvili G., Bezhanishvili N., Gabelaia D. and Kurz A.: Bitopological duality for distributive lattices and Heyting algebras. Mathematical Structures in Computer Science 20 (3), 359-393 (2010).

[3] Celani S. and Jansana R.: A closer look at some subintuitionistic logics. Notre Dame J. Formal Logic 42, 225-255 (2003).

[4] Celani S. and Jansana R.: Bounded distributive lattices with strict implication. Mathematical Logic Quarterly 51, 219-246 (2005).

[5] Cornish W.H.: On H. Priestley's dual of the category of bounded distributive lattices. Matematički Vesnik 12 (27) 60, 329-332 (1975).

[6] Davey B.A. and Priestley H.A.: Introduction to Lattices and Order. Cambridge Univ. Press (1994).

[7] Epstein G. and Horn A.: Logics which are characterized by subresiduated lattices. Mathematical Logic Quarterly 22 (1), 199-210 (1976).

[8] Fleisher I.: Priestley's duality from Stone's. Advances in Applied Mathematics 25 (3) 233-238 (2000).

[9] Priestley H.A.: Representation of bounded distributive lattice by means of orderer Stone spaces. Bulletin London Math. Soc. 2, 186-190 (1970).

[10] San Martín H.J.: Compatible operations in some subvarieties of the variety of weak Heyting algebras. In: Proceedings of the 8th Conference of the European Society for Fuzzy Logic and Technology (EUSFLAT 2013). Advances in Intelligent Systems Research, pp. 475-480. Atlantis Press (2013).

[11] Stone M.: Topological representation of distributive lattices and Brouwerian logics. Casopis pro pěstování matematiky a fysiky 67 (1), 1-25 (1938).

Sergio Arturo Celani,

Departamento de Matemática,

Facultad de Ciencias Exactas (UNCPBA),

Pinto 399, Tandil (7000),

and CONICET, Argentina,

scelani@exa.unicen.edu.ar

Agustín Leonel Nagy

Departamento de Matemática,

Facultad de Ciencias Exactas (UNCPBA), and CIC.

Pinto 399, Tandil (7000),

agustin.nagy@gmail.com

Hernán Javier San Martín, Departamento de Matemática, Facultad de Ciencias Exactas (UNLP), and CONICET.

Casilla de correos 172, 
La Plata (1900), Argentina.

hsanmartin@mate.unlp.edu.ar 